\title{
On Partial Weakly Clustering States with an Application to the Ising Model
}

\author{
G. G. EMCH \\ Department of Physics and Astronomy, The University of Rochester \\ H. J. F. KNops and E. J. Verboven \\ Instituut voor theoretische Fysika, Universiteit Nijmegen \\ Received February 2, 1968
}

\begin{abstract}
The concept of partial weak clustering in the mean is defined for states on physical systems which admit amenable (semi-)groups of symmetries. The properties of partial weak clustering states are studied and the relations between these states and (extremal) invariant (partial) states are considered. As an application we discuss the zero field magnetization in the two-dimensional Ising model.
\end{abstract}

\section{Introduction}

In a previous paper [1] we started an investigation of the properties of partial invariant states. We proved that a partial potentially invariant (resp. extremal invariant) state, defined on some appropriate subspace of a $C^{*}$-algebra can always be extended to an invariant (resp. extremal invariant) state on the whole algebra. Possible applications of the results to statistical mechanics were indicated.

In the present paper we consider weakly clustering partial states, instead of extremal invariant partial states. Our principal motivation in expanding our previous work in the present direction is that "partial weak clustering" seems to be a more appropriate condition than "extremal invariance of partial states" when one wants to select possible candidates for partial states that could be extended into extremal invariant states on the whole algebra. Our interest in this type of extension is, on the one hand, that in concrete applications "partial weak clustering" is much easier to prove than extremal (potential) invariance of partial states. On the other hand, extremal invariant states on the whole algebra have been proposed to be identified with pure thermodynamical phases; this proposal has the advantage to give a clear mathematical definition of the latter physical concept. The appropriateness of this definition will be illustrated in the application to the Ising (lattice gas) model which closes this paper. One appealing and general reason for this association between pure thermodynamical phases and extremal invariant states is the following: for asymptotically abelian algebras, it is known that on states on the whole algebra, the condition of extremal 
invariance is equivalent to the condition of weak clustering and, therefore, implies that the corresponding partial states are also weakly clustering. However the former condition does not imply that these partial states are extremal invariant. In fact, the condition of extremal (potential) invariance for partial states seems to be to drastic a requirement for physical purposes. All of these facts seem then to justify the terms in which we state the extension problem in this paper.

We first collect in Section 2 some of the basic facts pertinent to the study of physical systems which admit amenable symmetry (semi-) groups. The definition of partial $\eta$-weakly clustering states is opening Section 3 which is devoted to their study. We prove that the decomposition of an invariant state into states that are only weakly clustering on a subspace is unique. We next show that for each invariant state $\phi$, weakly clustering on a separable self-adjoint subspace $\mathfrak{M}$ of an asymptotically abelian algebra $\mathfrak{A}$, there exists at least one state $\phi^{\prime}$, weakly clustering on the whole algebra $\mathfrak{A}$ and which coincides with $\phi$ on $\mathfrak{M}$. In order to illustrate the abstract theory developed so far, we propose in Section 4 an application to the two dimensional Ising model. The spontaneous magnetization, defined originally as the residual magnetization in zero magnetic field, has been obtained by indirect computations in essentially three different ways $[2,3,4]$. It has been shown by SML [4] that all three of these derivations are equivalent to each other. SML further indicate that they are equivalent to the original definition only if one assumes that the probability density distribution of the magnetization is very sharply peaked around the values $\pm M_{0}$ when the thermodynamical limit is approached. Working on the properly infinite system, we take advantage of the general theory developed in Section 3 to argue that the agreement between the usual derivations and the original definition is indeed to be expected from the coexistence of two pure thermodynamical phases below the critical temperature.

\section{Invariant Means and Amenable (Semi-)groups}

This section is meant to serve the purpose of introducing the notation and directing the reader's attention to some facts pertinent to our study.

The notion of amenable (semi-)groups was formally introduced by DAY [5], although similar ideas were already latent in such earlier works as von NEUMANN's [6]. We call mean over a (semi-)group $G$, a positive, linear, normalized functional on the space $\mathfrak{E}(G)$ of all bounded, complex valued functions on $G$. The (semi-)group $G$ is said to be amenable if there exists a mean over $G$ which is invariant under all right and left translations by elements of $G$. If $G$ is furthermore equipped with a topology which makes it to be a topological (semi-)group, we impose in the 
definition above a further condition on $\mathfrak{S}(G)$, namely, that its elements are continuous on $G$. It might incidentally be noted that the amenability of a (semi-)group depends in an essential way on its topology. Also, it should be noticed that we do not take for $\mathfrak{C}(G)$ neither the space of almost periodic nor of ergodic functions since in this latter cases there always exists an invariant mean and, therefore, our definition of amenable (semi-)groups would then become redundant. For a general discussion of the properties of amenable groups, semi-groups and topological (semi-)groups, see, for instance, respectively, von Neumann [6], Day [7], and Dixmier [8], and, for later developments, Pier [9]. The relevance of the notion of amenability for systems occurring in Statistical Mechanics has been emphasized recently by Doplicher, Kadison, KaStLer and Robinson [10] (we shall use in the sequel the notation of [1]). We might mention here again that the translation group, the rotation group and the euclidian group in $R^{n}$, equipped with their usual topologies, are amenable. For the translation group in $R^{3}$ the invariant mean is nothing but the so-called Wiener mean

$$
\eta(f)=\lim _{R \rightarrow \infty} \frac{1}{\frac{4 \pi}{3} R^{3}} \int_{x} f(x) d^{3} \boldsymbol{x},
$$

whenever this limit exists; otherwise it is defined by extension.

In keeping with our previous paper [1] we say that a physical system $(\mathfrak{A}, \mathfrak{E})$, consisting of a $C^{*}$-algebra $\mathfrak{A}$ and its set of states $\mathfrak{E}$, admits $G$ as a symmetry if for every element $g$ in $G$ we are given an affine $w^{*}$ unimorphism $v_{g}$ of $\subseteq$ onto itself such that the "expectation values" $\left(v_{g} \phi\right)(A)$ are continuous functions on $G$ for all $(A, \phi)$ in $(\mathfrak{A}, \mathbb{E})$. This requirement has an immediate physical meaning. In spite of its apparent weakness, it is effectively a very strong one, as has been emphasized by KADISON [11]. In particular it implies that for any element $g$ of $G$ there exists a Jordan *-automorphism $\alpha_{g}$ of $\mathfrak{A}$ such that

$$
\left(v_{g} \phi\right)(A)=\phi\left(\alpha_{g} A\right) \text { for all }(A, \phi) \text { in }(\mathfrak{A}, \mathfrak{E}) .
$$

[By a Jordan *-automorphism we mean a linear, *-preserving mapping $\alpha$ of $\mathfrak{A}$ onto itself such that $\alpha(A B+B A)=\alpha(A) \alpha(B)+\alpha(B) \alpha(A)$ for all $A$ and $B$ in $\mathcal{Q}$.] This mapping $\alpha_{g}$ is evidently weakly continuous in $g$. This establishes the equivalence between the Schrödinger picture and the Heisenberg picture. The Heisenberg picture is sometimes presented in a stronger form, namely one requires that the $\alpha_{g}$ are $C^{*}$-automorphisms and not only Jordan *-automorphisms, i. e. that $\alpha_{g}(A B)=\alpha_{g}(A) \alpha_{g}(B)$. In general this requirement is hardly justifiable from direct physical arguments, but it might be of some convenience from the mathematical point of view. Without further physical requirements than those we imposed in our definition of $v_{g}$ there are however some interesting 
particular cases where this mathematical convenience is met anyhow, e. $g$. when $\mathfrak{A}$ is abelian or when $G$ is connected. We shall not need more on this equivalence in the sequel.

At this point it is useful to recall some results of KADIson [11] ( $\$ 2)$ and Dixmier [12] ( $\S \mathrm{XII}$ ). The universal representation $\pi_{u}$ of $\mathfrak{A}$ is defined as the direct sum of all cyclic representations $\pi_{\phi}$ of $\mathfrak{A}$. It is faithful; its weak closure $\pi_{u}(\mathfrak{U})^{\prime \prime}$ is called the enveloping von Neumann algebra of $\mathfrak{A}$ and we shall denote it by $\mathfrak{U}^{\prime \prime}$. There exists an isometric isomorphism $A$ from $\mathfrak{U}^{\prime \prime}$ onto the double dual $\mathfrak{A}^{* *}$ of $\mathfrak{A}$. This isomorphism transforms the weak-operator topology on $\mathfrak{A}^{\prime \prime}$ into the weak *-topology on $\mathfrak{A}^{* *}$, and $\Lambda \circ \pi_{u}$ is the canonical embedding of $\mathfrak{A}$ into $\mathfrak{A}^{* *}$. For any representation $\pi$ of $\mathfrak{A}$ there exists an unique normal (and hence ultraweakly continuous) representation $\tilde{\pi}$ extending $\pi$ from $\mathfrak{A}$ to $\mathfrak{A}^{\prime \prime}$, and one has $\tilde{\pi}\left(\mathfrak{U}^{\prime \prime}\right)=\pi(\mathfrak{U})^{\prime \prime}$. If $\alpha$ is a Jordan *automorphism (resp. a $C^{*}$-automorphism) of $\mathfrak{A}$, its (ultra) weakly continuous extension $\tilde{\alpha}$ to $\mathfrak{A}^{\prime \prime}$ is a Jordan *-automorphism (resp. a $C^{*}$-automorphism) of $\mathfrak{A}^{\prime \prime}$ and one has $\Lambda \tilde{\alpha} \Lambda^{-1}=\nu^{*}$, where $\nu^{*}$ is the adjoint of $\nu$. Every state $\phi$ on $\mathfrak{A}$ possesses a unique weakly continuous extension $\tilde{\phi}$ to $\mathfrak{A}^{\prime \prime}$.

If $\eta$ is a mean (resp. an invariant mean) over $G$, one verifies that for every $(A, \phi)$ in $(\mathfrak{A}, \mathfrak{S})$

$$
\eta\left\{\left(\nu_{g} \phi\right)(A)\right\}=(\eta \phi)(A)=\phi(\eta A)
$$

defines uniquely a state (resp. an invariant state) $(\eta \phi)$ in $\mathfrak{E}$, and an element (resp. an invariant element) $(\eta A)$ in $\mathfrak{X}^{* *}$. Hence in the particular case where $\mathfrak{A}$ is the algebra of quasi-local observables on a physical system, we have defined for every quasi-local observable $A$ an "averaged observable" $\eta A$ with respect to the action $g \rightarrow \nu_{g}$ of the symmetry group $G$. If for instance $t \rightarrow \nu_{t}$ is the prescription giving the evolution in time, $\eta A$ is what is traditionally meant by the "ergodic average" of $A$. In the particular case considered as an application in section 4 , if $\sigma_{i}^{z}$ denotes the spin attached to the site $i$ of the lattice, and $G$ is the discrete translation group of the lattice, $\eta \sigma_{i}^{z}$ gives a precise mathematical meaning to the observable expected to describe the magnetization of the Ising lattice considered.

The fact that $\eta A$ belongs to $\mathfrak{A} * *$ but not necessarily to $\mathfrak{A}$ implies that for a given representation $\pi$ of $\mathfrak{A}, \eta^{\pi}(A)$ defined as $\tilde{\pi} \circ \Lambda^{-1} \circ \eta(A)$ belongs to $\pi(\mathfrak{A})^{\prime \prime}$ but in general not to $\pi(\mathfrak{U})$. We notice that if $\pi_{\phi}$ is the cyclic representation generated from any state $\phi$, and $\Omega_{\phi}$ the corresponding cyclic vector, one has:

$$
\eta\left\{\boldsymbol{v}_{g} \phi(A)\right\}=\phi(\eta A)=\left(\Omega_{\phi}, \eta_{\phi}^{\pi}(A) \Omega_{\phi}\right) .
$$


If moreover $\alpha_{g}$ is unitarily implemented by $U_{\phi}(g)$ in the representation $\pi_{\phi}$ then $\eta_{\phi}^{\pi}(A)$ belongs to

$$
\left\{\pi_{\phi}(\mathfrak{R})\right\}^{\prime \prime} \cap\left\{U_{\phi}(G)\right\}^{\prime}
$$

but might still lay outside $\pi_{\phi}(\mathfrak{U})$ itself.

In closing this section we want to recall the following notion: a state $\phi$ on $\mathfrak{A}$ is said to be $\eta$-weakly clustering whenever:

$$
\phi(A \eta B)-\phi(A) \phi(\eta B)=0 \text { for all } A, B \in \mathfrak{A}
$$

where $\phi(A \eta B)$ is a short-hand notation for $\tilde{\phi}\left(\pi_{u}(A)\left(\Lambda^{-1} \circ \eta\right)(B)\right)$. Hence in the GNS representation generated by $\phi,(3)$ becomes:

$$
\left(\Omega_{\phi}, \pi_{\phi}(A) \eta_{\phi}^{\pi}(B) \Omega_{\phi}\right)=\left(\Omega_{\phi}, \pi_{\phi}(A) \Omega_{\phi}\right)\left(\Omega_{\phi}, \eta_{\phi}^{\pi}(B) \Omega_{\phi}\right) .
$$

In the Heisenberg picture (3) takes the form:

$$
\eta\left\{\phi\left(A \alpha_{g} B\right)\right\}=\phi(A) \eta\left\{\phi\left(\alpha_{g} B\right)\right\} .
$$

If $\phi$ is moreover invariant, formula (3) obviously reduces to

$$
\phi(A \eta B)-\phi(A) \phi(B)=0 \text { for all } A, B \in \mathfrak{A}
$$

and implies that $\phi$ is extremal invariant. The converse is also true (namely that extremal invariant states are $\eta$-weakly clustering), provided that the algebra $\mathfrak{A}$ satisfies a property, called $\eta$-asymptotic abelianness, which amounts to say that

$$
\Lambda^{-1} \circ \eta: \mathfrak{A} \rightarrow \pi_{u}(\mathfrak{A})^{\prime}
$$

and since we already know that the image of $\mathfrak{A}$ through $\Lambda^{-1} \circ \eta$ is contained in $\pi_{u}(\mathfrak{Q})^{\prime \prime},(5)$ implies that $\Lambda^{-1} \circ \eta$ maps $\mathfrak{A}$ into the center of $\mathfrak{A}^{\prime \prime}$. The physical meaning of this condition becomes clearer when it is realized that (5) is equivalent to:

$$
\eta_{\phi}^{\pi}: \pi_{\phi}(\mathfrak{A}) \rightarrow \pi_{\phi}(\mathfrak{Q})^{\prime} \text { for all states } \phi \text { on } \mathfrak{A} \text {. }
$$

Formula (4), which implies in particular the absence of long-range order, is believed to be a characteristic of pure phases; in this latter context it might be appropriate to recall that every invariant state can be decomposed in a unique way into $\eta$-clustering states.

For further details on the material reviewed in this section, the reader is referred to $[1,10,13,14]$.

\section{Partial Weakly Clustering States}

The definition of partial potentially invariant states on a self-adjoint subspace of a given algebra has been given in [1] where we indicated the practical interest of gathering information about these states. We concentrated, in that paper, on the concepts of invariance and extremal invariance with the principal result of proving that there always exists 
an invariant (respectively extremal invariant) extension $\phi$, to the whole of $\mathfrak{A}$, of any given potentially invariant (respectively extremal invariant) partial state $f$, defined initially only on a self-adjoint, vectorial subspace $\mathfrak{W}$ of $\mathfrak{A}$, containing the identity of $\mathfrak{A}$.

We now intend to see whether it is possible to carry over a similar program for the property of $\eta$-weakly clustering, to establish its relation to extremal potential invariance, and in general, to determine the extent to which the results mentioned in Section 2 remain valid for partial states as well.

Definition 1. Let $\mathfrak{M}$ be a subset of a $C^{*}$-algebra $\mathfrak{A}$. We say that a state $\phi$ on $\mathfrak{A}$ is $\eta$-weakly clustering on $\mathfrak{M}$ whenever

$$
\phi\left(M_{1} \eta M_{2}\right)=\phi\left(M_{1}\right) \phi\left(\eta M_{2}\right) \text { for all } M_{1}, M_{2} \in \mathfrak{R} \text {. }
$$

Lemma 1. Let $G$ amenable be a symmetry for the physical system ( $(\mathfrak{S})$, $\eta$ be an invariant mean over $G, \phi$ be $G$-invariant in $\mathcal{E}, \mathscr{H}$ be a Hilbert space, $\Omega$ a vector in $\mathscr{H}$ and $\pi$ be a representation of $\mathfrak{A}$ in the bounded operators of $\mathscr{H}$, such that

$$
(\Omega, \pi(A) \Omega)=\phi(A) \text { for all } A \in \mathfrak{A} .
$$

Then for any self-adjoint subspace $\mathfrak{M}$ of $\mathfrak{A}$ the following two conditions are equivalent:

(i) $\phi$ is $\eta$-weakly clustering on $\mathfrak{M}$.

(ii) $\Omega$ is an eigenvector of $\eta^{\pi}(M)$ for every $M$ in $\mathfrak{M}$.

If, furthermore, $\Omega$ is cyclic for $\pi(\mathfrak{Z})$ and $\mathfrak{A}$ is $\eta$-asymptotically abelian, condition (ii) above can be replaced by the equivalent condition.

(ii)' $\eta^{\pi}(M)$ is a c-number in $\mathscr{H}$.

Proof. Let us first prove $(\mathrm{i}) \rightarrow($ ii). Since $\phi$ is invariant, we have

$$
\left.\begin{array}{rl}
\phi(A \eta B) & =\phi(\eta A \cdot \eta B) \\
\phi(A) \phi(B) & =\phi(\eta A) \phi(\eta B)
\end{array}\right\} \text { for all } A, B \in \mathfrak{A} \text {. }
$$

Hence (i) is equivalent to

$$
\phi\left(\eta M_{1} \cdot \eta M_{2}\right)=\phi\left(\eta M_{1}\right) \phi\left(\eta M_{2}\right) \text { for all } M_{1}, M_{2} \in \mathfrak{W} \text {. }
$$

Passing now to the representation $\pi$, we recall that, as mentioned in section $2, \eta(M)$ is represented in $\mathfrak{Z}(\mathscr{H})$ by an element $\eta^{\pi}(M)$ of $\pi(\mathfrak{U})^{\prime \prime}$. Since $\mathfrak{M}$ is self-adjoint, we can write in particular $M_{1}=M=M_{2}^{*}$ in (8), which now implies in the representation considered:

$$
\left\|\left\{\eta^{\pi}(M)-\left(\Omega, \eta^{\pi}(M) \Omega\right)\right\} \Omega\right\|=0
$$

which is equivalent to (ii). The implication (ii) $\rightarrow$ (i) is proved along the same lines, using now that $\mathfrak{M}$ is moreover a subspace of $\mathfrak{A}$. The equivalence between (ii) and (ii)', in the case described in the statement of the lemma is seen as follows. From (5) we have, in particular, that $\eta^{\pi}(M)$ commutes with $\pi(A)$ for all $M$ in $\mathfrak{R}$ and all $A$ in $\mathfrak{A}$. 
Consequently,

$$
\eta^{\pi}(M) \pi(A) \Omega=\pi(A) \eta^{\pi}(M) \Omega
$$

and, since $\pi(A) \Omega$ is dense in $\mathscr{H},(10)$ leads directly to the proof of the equivalence between (ii) and (ii)'. This achieves the proof of lemma 1 .

Theorem 1. Let $G$ amenable be a symmetry for ( $\mathfrak{A}, \subseteq), ~ \eta$ be an invariant mean over $G, \phi$ be $G$-invariant in $\mathfrak{S}$ and $\mathfrak{M}$ be a self-adjoint subspace of $\mathfrak{A}$. If $\phi$ can be decomposed into a finite convex sum of invariant states $\phi_{i}$ which are $\eta$-weakly clustering on $\mathfrak{M}$ and such that their respective restrictions to $\mathfrak{M}$ are distinct from one another, then this decomposition is unique.

Proof. In order to set up the proof we need some particular properties of the assumed decomposition, which we want to prove first. Let us hence suppose that there exists at least one decomposition of $\phi$ satisfying the conditions of the theorem

$$
\phi=\sum_{i=1}^{N} \alpha_{i} \phi_{i} ; \quad 0<\alpha_{i}<1 ; \quad \sum_{i=1}^{N} \alpha_{i}=1 .
$$

Let $\pi$ be any representation of $\mathfrak{A}$ where $\phi$ is a vector state, $i$. e. such that

$$
\phi(A)=(\Omega, \pi(A) \Omega) \text { for all } A \in \mathfrak{A} .
$$

From (11) we have that $\alpha_{i} \phi_{i}$ is a positive linear form on $\mathfrak{A}$ majorized by $\phi$. We can hence use Proposition 2.5.1 in Dixmrer [12] to assert the existence of a self-adjoint operator $B_{i}$ belonging to the commutant of $\pi(\mathfrak{U})$ and such that:

$$
\phi_{i}(A)=\left(B_{i} \Omega, \pi(A) B_{i} \Omega\right) \text { for all } A \in \mathfrak{U} .
$$

Since $\phi_{i}$ is $\eta$-weakly clustering on $\mathfrak{M}$, we conclude from (13) and lemma 1 that $B_{i} \Omega$ is an eigenvector of $\eta^{\pi}(M)$ for every $M$ in $\mathfrak{M}$. Since further $B_{i}$ belongs to $\pi(\mathfrak{U})^{\prime}$ and $\eta^{\pi}(M)$ belongs to $\pi(\mathfrak{U})^{\prime \prime}, B_{i}$ commutes with $\eta^{\pi}(M)$ and, therefore, $B_{i}^{2} \Omega$ is also an eigenvector of $\eta^{\pi}(M)$, corresponding to the same eigenvalue as $B_{i} \Omega$. Let $\lambda_{i}(M)$ be this eigenvalue. Since the restrictions to $\mathfrak{W}$ of the $\phi_{i}$ 's are distinct from one another by assumption, we have for any pair $(i, j)$ with $i \neq j$, at least one self-adjoint element $M_{i j}$ in $\mathfrak{M}$ such that

$$
\phi_{i}\left(M_{i j}\right) \neq \phi_{j}\left(M_{i j}\right) .
$$

Since $\phi_{i}, \phi_{j}$ are invariant, we have as well

and, consequently,

$$
\phi_{i}\left(\eta M_{i j}\right) \neq \phi_{j}\left(\eta M_{i j}\right)
$$

$$
\left(B_{i} \Omega, \eta^{\pi}\left(M_{i j}\right) B_{i} \Omega\right) \neq\left(B_{j} \Omega, \eta^{\pi}\left(M_{i j}\right) B_{j} \Omega\right)
$$

and, since $B_{i} \Omega$ is an eigenvector of $\eta^{\pi}\left(M_{i j}\right)$ with eigenvalue $\lambda_{i}\left(M_{i j}\right)$, we get:

$$
\lambda_{i}\left(M_{i j}\right)\left(B_{i} \Omega, B_{i} \Omega\right) \neq \lambda_{j}\left(M_{i j}\right)\left(B_{j} \Omega, B_{j} \Omega\right)
$$


and hence:

since

$$
\lambda_{i}\left(M_{i j}\right) \neq \lambda_{j}\left(M_{i j}\right)
$$

$$
1=\phi_{i}(I)=\left(B_{i} \Omega, B_{i} \Omega\right) \text { for all } i .
$$

Consequently, $B_{i}^{2} \Omega$ and $B_{j}^{2} \Omega$ are orthogonal to one another since they belong to different eigenvalues of the self-adjoint operator $\eta^{\pi}\left(M_{i j}\right)$. If now $\Omega$ is cyclic for $\pi$ (e. g., if $\pi$ is the GNS representation $\pi_{\phi}$ ), then an immediate consequence of (11), (12), (13), and the above stated result is that the following decomposition of $\Omega$ in orthogonal vectors holds

$$
\Omega=\sum_{i=1}^{N} \alpha_{i} B_{i}^{2} \Omega .
$$

By this argument we reduced our problem to the proof of the uniqueness of the decomposition (14). Suppose then that we have another decomposition of $\phi$ :

$$
\phi=\sum_{j=1}^{M} \beta_{j} \psi_{j},
$$

satisfying the same condition as the decomposition (11). Let

$$
\Omega=\sum_{j=1}^{M} \beta_{j} D_{j}^{2} \Omega
$$

be the corresponding decomposition of $\Omega$. If on $\mathfrak{M}$ all the $\psi_{j}$ 's were different from all the $\phi_{i}$ 's, we would get by the same reasoning as before that all the $B_{i}^{2} \Omega$ were orthogonal to all the $D_{j}^{2} \Omega$, which is impossible since (14) and (16) are two decompositions in orthogonal vectors of the same vector $\Omega$. Hence at least one of the $\psi_{j}$ 's, which we call $\psi_{1}$, should coincide on $\mathfrak{M}$ with one of the $\phi_{i}$ 's, which we call $\phi_{1}$. And, then, since all the $\phi_{i}$ 's are different from one another on $\mathfrak{M}$, we have

$$
\left.\psi_{1}\right|_{\mathfrak{m}} \neq\left.\phi_{i}\right|_{\mathfrak{m}} \text { for all } i \neq 1 \text {, }
$$

and, consequently, $D_{1}^{2} \Omega$ is orthogonal to all $B_{i}^{2} \Omega$ with $i \neq 1$. Upon comparison of (14) and (16), we get that $D_{1}^{2} \Omega$ is proportional to $B_{1}^{2} \Omega$. The proportionality constant is indeed 1 as one realizes immediately by computing the value of $\phi_{1}(I)$ and $\psi_{1}(I)$ using (13), (14) and (16). We get $\alpha_{1}=\beta_{1}$ upon comparison of (14) and (16). We can then proceed by successive iterations and prove in the same way

$$
N=M ; \quad \alpha_{i}=\beta_{i} ; \quad \phi_{i}=\psi_{i} \text { for all } i
$$

which concludes the proof of the theorem.

We may notice that in the particular case where $\mathfrak{M}$ coincides with $\mathfrak{A}$ the uniqueness asserted in the above theorem has been established earlier. Much more, indeed, is known in that special situation, as can be 
found for instance in $[10,15]$, and, for earlier results, in $[13,14]$, as well as in $[16,17]$. The aim pursued here is to see to which extent some of the results previously obtained, can be generalized to partial states, that is, when conditions such as $\eta$-weak clustering are weakened so as to be imposed only on a subspace $\mathfrak{M}$ rather than on the whole algebra $\mathfrak{A}$. One step in this direction is that, as the result of the preceding theorem, we can generalize an assertion found, for instance, in [15], namely, that the decomposition of invariant states into weakly clustering states, which is interpreted as a decomposition of any equilibrium state into pure thermodynamical phases, should be unique for physical reasons, and actually is as has been proved, even if phases are identified through partial $\eta$-weak clustering. In this connection one should notice that, whereas $\eta$-weak clustering on $\mathfrak{A}$ implies extremal invariance, this is no more true for partial $\eta$-weak clustering on a subspace $\mathfrak{M}$ only; this latter property does not guarantee extremal invariance on $\mathfrak{A}$ nor even on $\mathfrak{M}$. The best we will be able to say in this respect is that when $\phi$ is $\eta$-weakly clustering on $\mathfrak{M}$, its restriction to $\mathfrak{M}$ admits an extremal invariant extension to $\mathfrak{A}$, provided that some further weak conditions on $\left(\mathfrak{A}, \mathbb{E}, \boldsymbol{v}_{g}\right)$ are satisfied. This is actually the assertion of the next theorem. While we are considering this type of question related to the connection between extremal invariance and weak clustering, we might recall that for an $\eta$-asymptotically abelian algebra $\mathfrak{A}$, extremal invariance of a state implies $\eta$-weak clustering, and, consequently, partial $\eta$-weak clustering on any subspace $\mathfrak{M}$ of $\mathfrak{A}$.

Lemma 2. Let $G$ amenable be a symmetry for $(\mathfrak{A}, \mathbb{E}), \eta$ be an invariant mean over $G, \phi$ and $\psi$ be $G$-invariant in $\mathfrak{S}$ and $\mathfrak{M}$ be a self-adjoint subspace of $\mathfrak{A}$. Suppose further that $\phi$ is $\eta$-weakly clustering on $\mathfrak{M}$ and that $\psi \leqq \lambda \phi$ for some positive real number $\lambda$. Then $\psi$ is also $\eta$-weakly clustering on $\mathfrak{M}$ and coincides with $\phi$ on $\mathfrak{R}$.

Proof. Let $\pi$ be a representation (e. g., the GNS representation) of $\mathfrak{A}$ for which $\phi$ is a vector state, i. e.,

$$
\phi(A)=(\Omega, \pi(A) \Omega) .
$$

From proposition 2.5.1 in Dixmier [12], we know that there exists a (selfadjoint, positive) element $B$ in $\pi(\mathfrak{Q})^{\prime}$ such that

$$
\psi(A)=(B \Omega, \pi(A) B \Omega) .
$$

We first notice that $\|B \Omega\|=1$ [since $\psi(I)=1$ ], that $B$ commutes also with $\eta^{\pi}(A)$ for all $A$ in $\mathfrak{A}$ and that $\Omega$ is an eigenvector of $\eta^{\pi}(M)$ for all $M$ in $\mathfrak{M}$ (by lemma 1). Consequently, $B \Omega$ is also an eigenvector of $\eta^{\pi}(M)$, corresponding to the same eigenvalue as $\Omega$ does. We have then

$$
\begin{aligned}
\psi(\eta M) & =\left(B \Omega, \eta^{\pi}(M) B \Omega\right) \\
& =\left(\Omega, \eta^{\pi}(M) \Omega\right)=\phi(\eta M)
\end{aligned}
$$


and since $\psi$ and $\phi$ are invariant, this proves the second assertion of the lemma. Furthermore, we see immediately, upon writing it in the representation space, that the following equality is true for any $A$ in $\mathfrak{A}$ and any $M$ in $\mathfrak{R}$ :

$$
\psi(A \eta M)=\psi(A) \psi(\eta M)
$$

which concludes the proof of the lemma. Incidentally, it follows from lemma 1 that (18) is actually no more general than (6).

We now use the preceeding lemma to prove the following theorem which might, actually, be stated in a more general way. To keep both the proof and the technical requirements to a reasonable length, we however chosed to compromize between the generality maintained throughout this section and the more specific needs of the next section.

Theorem 2. Let $G$ amenable be a symmetry for $(\mathfrak{A}, \mathfrak{S}), \eta$ be an invariant mean over $G, \mathfrak{M}$ be a self-adjoint subspace of $\mathfrak{A}$, and $\phi$ be $G$-invariant in $\mathfrak{S}$ and $\eta$-weakly clustering on $\mathfrak{M}$. We suppose further that: (i) $\mathfrak{Z}$ is $\eta$-asymptotically abelian and $G$ is connected, or ( $\left.\mathrm{i}^{\prime}\right) \mathfrak{A}$ is (weakly) asymptotically abelian; (ii) $\nu_{g}$ is implemented by a homomorphism $g \rightarrow \alpha_{g}$ from $G$ to the group of $C^{*}$-automorphisms of $\mathfrak{A}$; and (iii) $\mathfrak{A}$ is separable. Then, the restriction $\left.\phi\right|_{\mathfrak{2}}$ of $\phi$ to $\mathfrak{M}$ admits an extremal G-invariant extension to $\mathfrak{A}$.

Proof. Let $\pi_{\phi}$ be the representation of $\mathfrak{A}$ induced by $\phi, \Omega_{\phi}$ be the corresponding cyclic vector and $\mathscr{H}_{\phi}$ be the representation space. Condition (ii) insures that there exists an unique continuous representation $U_{\phi}$ of $G$ such that

$$
\left.\begin{array}{rl}
\pi_{\phi}\left(\alpha_{g}(A)\right) & =U_{\phi}(g) \pi_{\phi}(A) U_{\phi}(g)^{-1} \\
\pi_{\phi}\left(\alpha_{g}(A)\right) \Omega_{\phi} & =U_{\phi}(g) \pi_{\phi}(A) \Omega_{\phi}
\end{array}\right\} \text { for all } A, g \text { in } \mathfrak{A}, G .
$$

Let $R_{\phi}$ be the von Neumann algebra generated in $\mathscr{H}_{\phi}$ by $\pi_{\phi}(\mathfrak{A})$ and $U_{\phi}(G)$. From either condition (i) or (i') we can then conclude, using the analysis of ref. [10], that $R_{\phi}^{\prime} \subseteq \pi_{\phi}(\mathfrak{A})^{\prime \prime}$, and therefore that $R_{\phi}^{\prime}$ is the set of all elements in the center of $\pi_{\phi}(\mathfrak{U})^{\prime \prime}$ which commute with $U_{\phi}(g)$ for all $g$ in $G$. In particular this implies that $R_{\phi}^{\prime}$ is abelian. In the particular case where $R_{\phi}^{\prime}$ is moreover generated by its minimal projections, we know [10] that $\phi$ can be decomposed (in a unique way) into a convex sum of extremal invariant (and hence $\eta$-weakly clustering) states on $\mathfrak{A}$. Each of these states $\psi_{\lambda}$ is majorized by $\phi$ (i. e. $\psi_{\lambda} \leqq p_{\lambda} \phi$ for some finite positive number $p_{\lambda}$ ). We can hence use our lemma 2 to conclude that the $\psi_{\lambda}$ and $\phi$ coincide on $\mathfrak{2}$. This concludes the proof in this particular case. In the more general case where $R_{\phi}^{\prime}$ is not necessarily generated by its minimal projections, our assumption (iii) ensures that $\mathscr{H}_{\phi}$ is separable, and then the above discrete sum becomes in general a "convex" integral of extremal invariant states:

$$
\phi=\int_{\Lambda} d \mu(\lambda) \psi_{\lambda}
$$


which means

$$
\phi(A)=\int_{A} d \mu(\lambda) \psi_{\lambda}(A) \text { for all } A \text { in } \mathfrak{A}
$$

and is unique $\left(R_{\phi}^{\prime} \leqq \pi_{\phi}(\mathfrak{U})^{\prime \prime}\right.$ is used in that part of the proof [10]). To conclude the proof in the general case considered now, it remains to see that at least one of the $\psi_{\lambda}$ coincides with $\phi$ on $\mathfrak{M}$. Let $E$ be a measurable subset of $\Lambda$ and let us define the following invariant state on $\mathfrak{A}$ :

$$
\psi_{E}=\frac{1}{\mu(E)} \int_{E} d \mu(\lambda) \psi_{\lambda} .
$$

$\psi_{E}$ is majorized by $\phi$ and hence, by lemma 2 again, coincides with $\phi$ on $\mathfrak{M}$. Consequently for every measurable set $E$ in $A$, we have:

$$
\int_{E} d \mu(\lambda)\left\{\psi_{\lambda}(M)-\phi(M)\right\}=0 \text { for all } M \text { in } \mathfrak{R}
$$

which implies for each $M$ separately:

$$
\psi_{\lambda}(M)=\phi(M) \text { for } \mu \text {-almost all } \lambda \text {. }
$$

Since $\mathfrak{A}$ is separable, so is $\mathfrak{M}$; hence the set

$$
\left\{\lambda \in \Lambda\left|\psi_{\lambda}\right|_{\mathfrak{m}} \neq\left.\phi\right|_{\mathfrak{m}}\right\}
$$

is of measure zero, i. e. $\psi_{\lambda}$ coincides with $\phi$ on $\mathfrak{M}$ for $\mu$-almost all $\lambda$. This is all what is needed for the completion of the proof of the theorem.

\section{The Spontaneous Magnetization of a Two-dimensional Ising-Model}

We consider the usual two-dimensional Ising model for ferromagnetism, which consists of a "rectangular" lattice

$$
\{(m, n) \mid 1 \leqq m \leqq M ; 1 \leqq n \leqq N\}
$$

of "classical spins" $\sigma_{m, n}^{z}$ interacting with their nearest neighbors and with an external magnetic field $\mathscr{H}$ so that the total hamiltonian of the system is :

$$
\left.\begin{array}{rl}
H_{M, N}(\mathscr{H})= & -\mathscr{H} \sum_{m, n} \sigma_{m, n}^{z} \\
& -J_{1} \sum_{m, n} \sigma_{m, n}^{z} \sigma_{m+1, n}^{z} \\
& -J_{2} \sum_{m, n} \sigma_{m, n}^{z} \sigma_{m, n+1}^{z}
\end{array}\right\}
$$

The spontaneous magnetization is defined as:

with

$$
\mathfrak{M}_{0}(\beta)=\lim _{\mathscr{H} \rightarrow 0^{+}} \lim _{M, N \rightarrow \infty} \mathfrak{M}_{M, N}(\beta, \mathscr{H})
$$

$$
\mathfrak{M}_{M, N}(\beta, \mathscr{H})=\left\langle\frac{1}{M N} \sum_{m, n} \sigma_{m, n}^{z}\right\rangle_{\beta, \mathscr{H}}
$$


where \langle\rangle$_{\beta, \mathscr{H}}$ denotes the canonical ensemble average in the magnetic field $\mathscr{H}$ and at the temperature $T=(k \beta)^{-1}$. To date, the canonical partition function, and hence the corresponding density matrix, have escaped all attempts to an exact solution, except in the particular case where $\mathscr{H}=0$. Since the two limits in (25) are not interchangeable, one has to find some round-about way to compute an exact expression for $\mathfrak{M}_{0}(\beta)$.

Montroll, Potts and WARD [2] and YANG [3] have proposed two different methods to accomplish this; they actually use alternative definitions of $\mathfrak{M}_{0}(\beta)$, namely ${ }^{1}$ :

$$
\begin{gathered}
\mathfrak{M}_{0}^{\text {MPW }}(\beta)=\left\{\lim _{m^{\prime}-m \rightarrow \infty} \lim _{M, N^{\prime} \rightarrow \infty}\left\langle\sigma_{m^{\prime}, n}^{z} \sigma_{m, n}^{z}\right\rangle_{\beta, 0}\right\}^{1 / 2}, \\
\mathfrak{M}_{0}^{\text {Yang }}(\beta)=\left|\lim _{\alpha \rightarrow 0^{+}} \lim _{\substack{M, N \rightarrow \infty \\
\mathscr{H}=\alpha / M}} \mathfrak{M}_{M, N}(\beta, \mathscr{H})\right| .
\end{gathered}
$$

Both of these formulas, when worked out, give the same result, namely that produced by Onsager [19]. Schultz, Matwis and Lieb [4] however emphasized that neither (27) nor (28) were ever rigorously proven to be equivalent to (25), as both MPW and Yang are forced to make extra assumptions that have not been justified, yet, on purely statistical mechanical grounds. SML show that these assumptions can be reduced to the supposition that the function $P_{M, N}(\mathfrak{M})$ (which is the probalitity density that the magnetization has the value $\mathfrak{M}$ in the absence of magnetic field) is very sharply peaked around the values $\pm \mathfrak{M}_{0}(\beta)$ in the thermodynamical limit. With this assumption, they justify a third definition of the spontaneous magnetization, which they prove to be equivalent to (27) and (28). Their definition reduces to formula (33) below.

Our aim in this section is to illustrate the general results of the previous sections in providing further evidence that the actual spontaneous magnetization (25) satisfies SML's definition and is hence given by Onsager's famous formula $[2,3,4]$.

Let $\mathfrak{A}_{M}$ be the $C^{*}$-algebra generated by

in the Hilbert space

$$
\left\{\sigma_{m, n}^{z} \mid 1 \leqq m \leqq M\right\}
$$

$$
\mathscr{H}_{M}=\bigotimes_{m} \mathscr{H}_{m, n}
$$

where $\mathscr{H}_{m, n}$ is the two-dimensional space relative to the site $(m, n)$. (From now on, we shall drop the index $n$, being understood that every statement made below is valid for any row.) The expectation value of any element $A$ in $\mathfrak{A}_{M}$, calculated for the canonical distribution is given, when $\mathscr{H}=0$, by

$$
\langle A\rangle_{M, N}(\beta)=\operatorname{Tr} A \varrho_{M}
$$

1 In this expression we use the equality between long-long range order and short long range as proven by SML [4]. 
with

$$
\varrho_{M}=\left(V_{M}\right)^{N} / \operatorname{Tr}\left(V_{M}\right)^{N}
$$

where $V_{M}$ is the usual transfer matrix. Below the critical temperature, we know from Onsager that a two-fold asymptotic degeneracy of the maximum eigenvalue of $V_{M}$ appears as $M \rightarrow \infty$. Let $\Psi_{M}^{+}$and $\Psi_{\bar{M}}^{-}$be the two corresponding eigenvectors of $V_{M}$. As a consequence of this degeneracy, (29) becomes in the thermodynamical limit:

$$
\begin{aligned}
\langle A\rangle_{\beta} & =\frac{1}{2} \lim _{M \rightarrow \infty}\left\{\psi_{M}^{+}(A)+\psi_{M}^{-}(A)\right\} \\
& =\frac{1}{2} \lim _{M \rightarrow \infty}\left\{\phi_{M}^{+}(A)+\phi_{M}^{-}(A)\right\}
\end{aligned}
$$

with

$$
\left.\begin{array}{l}
\psi_{M}^{ \pm}(A)=\left\langle\Psi_{\bar{M}}^{ \pm}|A| \Psi_{\bar{M}}^{ \pm}\right\rangle \\
\phi_{M}^{ \pm}(A)=\left\langle\Phi_{\bar{M}}^{ \pm}|A| \Phi_{M}^{ \pm}\right\rangle \\
\Phi_{M}^{ \pm}=\frac{1}{\sqrt{2}}\left(\Psi_{M}^{+} \pm \Psi_{\bar{M}}^{+}\right)
\end{array}\right\}
$$

in which the phases of $\Psi_{M}^{+}$and $\Psi_{\bar{M}}^{-}$are chosen in such a way that $\left\langle\Psi_{M}^{+}\left|\sigma_{m}^{z}\right| \Psi_{M}^{-}\right\rangle$is real and positive.

Consider now the $C^{*}$-algebra $\mathfrak{A}$ (obtained as the norm-closure of the union of all $\mathfrak{A}_{M}$ referring to the same row) and the states on $\mathfrak{A}$ defined by:

$$
\left.\begin{array}{l}
\psi^{ \pm}(A)=\lim _{M \rightarrow \infty} \psi_{M}^{ \pm}(A) \\
\phi^{ \pm}(A)=\lim _{M \rightarrow \infty} \phi_{\frac{ \pm}{M}}^{ \pm}(A)
\end{array}\right\} \text {. }
$$

From the well-known properties of the transfer matrix one has that the states $\psi^{ \pm}$and $\phi^{ \pm}$are invariant under the group $Z$ of the discrete translations along the row considered; moreover the phase of the flip-flop symmetry (reversing all spins) can be chosen in such a way that it changes $\Psi_{\vec{M}}^{ \pm}$into respectively $\pm \Psi_{\vec{M}}^{ \pm}$and consequently exchanges $\phi^{ \pm}$ into one another. For completeness let us recall here the third alternative form of the spontaneous magnetization (namely that of SML mentionned above):

$$
\left.\begin{array}{rl}
\mathfrak{M i}_{0}^{\mathrm{SML}}(\beta) & =\lim _{M \rightarrow \infty}\left\langle\Psi_{M}^{+}\left|\frac{1}{M} \Sigma \sigma_{m}^{z}\right| \Psi_{M}^{-}\right\rangle \\
& =\lim _{M \rightarrow \infty}\left\langle\Psi_{M}^{+}\left|\sigma_{m}^{z}\right| \Psi_{\bar{M}}^{-}\right\rangle
\end{array}\right\} .
$$

Let us now analyze the structure of the states so far obtained, in relation with the previous sections. From the discussion of SML we learn that:

$$
\begin{aligned}
\lim _{m^{\prime}-m \rightarrow \infty} & \lim _{M \rightarrow \infty} \frac{1}{2}\left\{\left\langle\Psi_{M}^{+}\left|\sigma_{m^{\prime}}^{z} \sigma_{m}^{z}\right| \Psi_{M}^{+}\right\rangle+\left\langle\Psi_{M}^{-}\left|\sigma_{m^{\prime}}^{z} \sigma_{m}^{z}\right| \Psi_{M}^{-}\right\rangle\right\} \\
= & \lim _{M \rightarrow \infty}\left\langle\Psi_{M}^{+}\left|\sigma_{m^{\prime}}^{z}\right| \Psi_{M}^{-}\right\rangle\left\langle\Psi_{M}^{-}\left|\sigma_{m}^{z}\right| \Psi_{M}^{+}\right\rangle
\end{aligned}
$$


from which we conclude (using the above definitions and the symmetry properties already mentioned):

and

$$
\lim _{m^{\prime}-m \rightarrow \infty}\left|\phi^{ \pm}\left(\sigma_{m^{\prime}}^{z} \sigma_{m}^{z}\right)-\phi^{ \pm}\left(\sigma_{m^{\prime}}^{z}\right) \phi^{ \pm}\left(\sigma_{m}^{z}\right)\right|=0
$$

$$
\phi^{+}\left(\sigma_{m}^{z}\right)=-\phi^{-}\left(\sigma_{m}^{z}\right)=\mathfrak{M}_{0}^{\mathrm{SML}}(\beta) \neq 0 .
$$

We then notice that $\phi^{+}$and $\phi^{-}$are clustering (and hence $\eta$-weakly clustering) on $\mathfrak{M}$, the subspace of all linear combinations of $\sigma_{m}^{z}$ 's and $I$. Moreover, when restricted to $\mathfrak{M}$ these two states are different. Hence the decomposition $\left(30^{\prime}\right)$ satisfies the assumptions of Theorem 1 and is therefore unique of its kind in the sense of the theorem. Furthermore, by theorem 2 we know that the states $\left.\phi^{+}\right|_{\mathfrak{m}}$ and $\left.\phi^{-}\right|_{\mathfrak{m}}$ can be extended to extremal invariant states on $\mathfrak{A}$, which are then interpreted as pure phases since they do not exhibit "long-range order". Let us now define the magnetization as the non-local observable:

$$
\eta \sigma_{m}^{z}=\lim _{M \rightarrow \infty} \frac{1}{M} \sum \sigma_{m}^{z}
$$

From lemma 1 , this is a $c$-number in the representation constructed on either of these states, and from (35) we see that this $c$-number takes precisely the value predicted by Onsager.

Hence the Onsager value for the spontaneous magnetization now appears as (the absolute value of) the expectation value of a macroscopic, intensive observable, the "magnetization" defined by (36), this expectation value being calculated on either of two extremal states, or pure phases, which, when restricted to $\mathfrak{R}$, decompose (see $30^{\prime}$ ) the equilibrium state \langle\rangle$_{\beta}$, defined by (30) and obtained by direct evaluation of the canonical distribution in the absence of magnetic field. The relevance of the existence and uniqueness of the decomposition $\left(30^{\prime}\right)$ appears in an even more familiar light when one considers the lattice-gas analog [20] of the two-dimensional Ising model. In this lattice-gas model, the decomposition $\left(30^{\prime}\right)$ corresponds to the decomposition of a mixture state (inside the coexistence region) into its pure phase components: the liquid and the gas. Incidentally, the fact that $\phi^{+}$and $\phi^{-}$are actually identified as pure phases on $\mathfrak{Z}$ only (since we only established that they are $\eta$ weakly clustering on $\mathfrak{W}$ ) is in agreement with the fact that the "equation of state" is actually a relation between the external contraints on the system (temperature and magnetic field) and an internal macroscopic observable in $\mathfrak{M}$, namely the magnetization (36).

Acknowledgements. We are indebted to Professor W. F. EBERLEIN who brought to our knowledge most of the quoted literature on amenable semi-groups. One of us (GGE) wants also to express his appreciation to the Faculteit der Wiskunde en Natuurwetenschappen, Katholieke Universiteit, Nijmegen for the hospitality extended to him during the Summer 1967. 


\section{Bibliography}

1. Ench, G. G., H. J. F. Knops, and F. J. Verboven: Commun. Math. Phys. 7 , $164-172(1968)$.

2. Montroll, E. W., R. B. Potrs, and J. C. Ward: J. Math. Phys. 4, 308 (1963).

3. YaNG, C. N.: Phys. Rev. 85, 808 (1952).

4. Schultz, T. D., D. C. Mattis, and E. H. Lieb : Rev. Mod. Phys. 36, 856 (1964).

5. Day, M. M.: Bull. Am. Math. Soc. 55, 1054 (1949).

6. von Neumann, J.: Fundamenta Math. 13, 73 (1929); Compositio Math. 1, 106 (1934).

7. DAY, M. M.: Illinois J. Math. 1, 509 (1957).

8. Dixmier, J.: Acta Sci. Math. Szeged 12, Pars A, 213 (1950).

9. Pier, J. P.: Thèse de 3 ième cycle, Nancy 1965.

10. Doplicher, S., R. V. Kadison, D. Kastler, and D. W. Robinson: Commun. Math. Phys. 6, 101 (1967).

11. Kadison, R. V.: Topology 3, Suppl. 2, 177 (1965).

12. Dixmier, J.: Les $C^{*}$-algèbres et leurs représentations. Paris: Gauthier Villars 1964.

13. Kastler, D., and D. W. Robinson: Commun. Math. Phys. 3, 151 (1966).

14. Ruelle, D.: Commun. Math. Phys. 3, 133 (1966).

15. Lanford, O., and D. Ruelle: J. Math. Phys. 8, 1460 (1967).

16. Doplicher, S., D. Kastler, and D. W. Robinson: Commun. Math. Phys. 3, 1 (1966).

17. Robinson, D., and D. Ruelle: Extremal invariant states, preprint IHES, 1966.

18. Dunford, N., and J. T. Schwartz: Linear operators. New York: Interscience Publ. 1958.

19. For an account of the esoteric character of Onsager's revelation the reader is referred to the introduction of MPW [2].

20. Lee, T. D., and C. N. YANG: Phys. Rev. 87, 410 (1952).

Dr. G. G. EMCH

Department of Physics and Astronomy

University of Rochester

Rochester, N. Y. 14627, USA 